\title{
Gallstone ileus, volvulus and intestinal perforation in a patient with cholecystostomy and choledochoduodenostomy
}

\begin{abstract}
Context: Gallstone ileus is an uncommon cause of small bowel obstruction with potentially serious complications including perforation and gangrene of the small bowel. Its diagnosis is difficult and surgery remains the mainstay of treatment. Here the complications of this entity are reported.

Case Report: A case is presented of small bowel obstruction owing to large gallstones
in terminal ileum in a 40 -year-old female patient. The diagnosis was made by computed
tomography and confirmed on exploratory laparotomy.

Conclusion: Although it is a rare cause of bowel obstruction, gallstone ileus should be capped in mind when a case of small bowel obstruction is dealt with because of its potentially serious complications. Early surgical management is the mainstay of treatment.
\end{abstract}

Keywords: gallstoneileus, volvulus, intestinal perforation, choledochoduodenostomy, seroperitoneum, laparotomy, pneumobilia
Volume 3 Issue I - 2017

\author{
Dongdong Wang, Yayuan Wei, Xiangbai Wu \\ Three Gorges University Colorectal Disease Research Institute, \\ China
}

Correspondence: Yayuan Wei, The Second Hospital of Yichang City, Three Gorges University Colorectal Disease Research Institute, No 21 Xiling First Street, Yichang 443000, China, Email1017440679@qq.com

Received: November 30, 2016 | Published: January 27, 2017

\section{Introduction}

Gallstone ileus first described by Bartolin in 1654 is an uncommon condition which may cause serious complications such as volvulus or intestinal perforation. There have been very few case reports about this because of its rarity. Here a case of a 40year-old female patient is reported, who presents with signs of small bowel obstruction owing to large gallstones in lower ileum with the serious complications of volvulus and intestinal perforation which has not been described in the last literature.

\section{Case description}

A female patient of 40 was admitted to our hospital with symptoms of intermittent abdominal distension, vomiting gastric contents and progressive abdominal pain; exhaust and defecation stopped for twenty days and the symptoms were aggravated in the last 2days. In her past medical history, she had undergone Cholecystostomy and Choledochoduodenostomy in 1996 and Bile duct exploration surgery due to common bile duct stones with acute choledochitis in 2002 . Plain abdominal X-ray demonstrated ileus and B-ultrasonic wave demonstrated stones in intra hepatic bile duct. The diagnosis of the local hospital was the possibility of extra hepatic bile duct expansion and calculus and ascites. With conservative treatment, the symptoms persist. Then a Computed Tomography scan in a certain municipal hospital demonstrated small bowel wall thickening with gut cavity expansion with the possibility of volvulus and small intestine blood supply obstacles, seroperitoneum and pelvic effusion. With conservative treatment, the symptoms remitted.

On physical examination, the female patient was found to have a distended, painful abdomen and weak bowel sounds with two operation scars at the right upper quadrant abdomen. She was a febrile and had a blood pressure of $100 / 70 \mathrm{mmHg}$, The Computed Tomography scan in our hospital demonstrated ileus with abdominal intestinal canal obviously effusion plot gas, intrahepatic bile ducts dilation and pneumobilia, hepatolithiasis, seroperitoneum, intestinal wall edema, with the possibility of stone obstruction in the small intestine (Figure 1). The gastrointestinal radiography using iohexol demonstrated duodenal diverticulum, intrahepatic bile ducts pneumobilia and ileus. On laparotomy, two large $(3 \mathrm{~cm})$ gallstones were identified obstructing the distal ileum and two perforations of size $7-8 \mathrm{~mm}$ and $2-3 \mathrm{~mm}$ respectively with approximately 1 meter of gangrenous patches in the adjacent bowel (Figure 3). The ileum $20 \mathrm{~cm}$ from the ileocecal junction twisted for $540^{\circ} \mathrm{C}$ (Figure 2). Resection anastomosis was done. The patient is recouping well postoperatively.

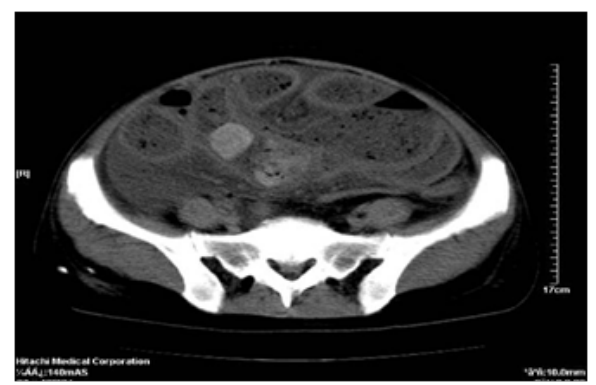

Figure I Computed Tomography scan revealed ileus, intrahepatic bile ducts dilation and pneumobilia, hepatolithiasis, seroperitoneum, Intestinal wall edema, stone obstruction in the small intestine. 

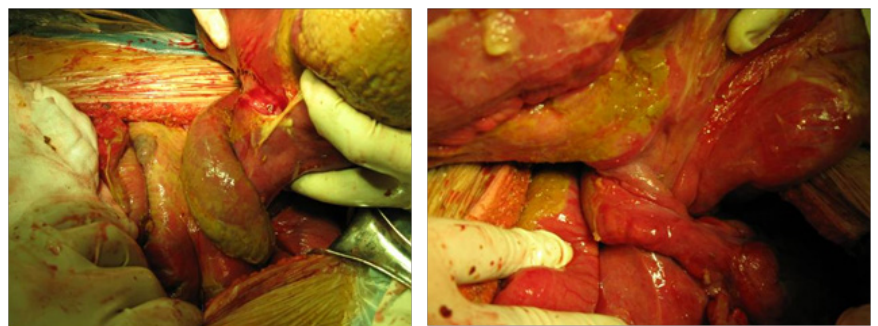

Figure 2 Intraoperative picture showing the ileum of $20 \mathrm{~cm}$ from the ileocecal junction volvulus of $540^{\circ} \mathrm{C}$.
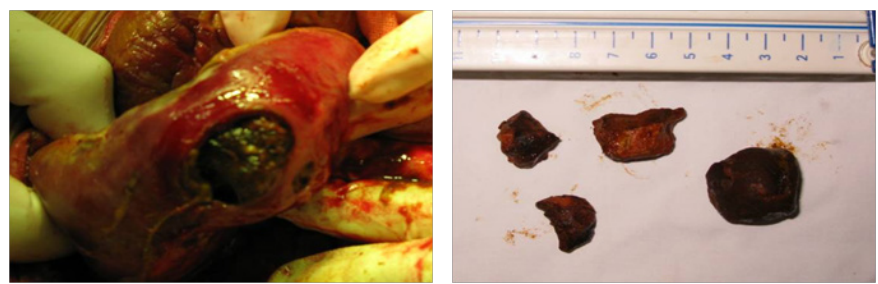

Figure 3 intraoperative pictures showing two large $(3 \mathrm{~cm})$ gallstones obstructing the distal ileum and two perforations of size $7-8 \mathrm{~mm}$ and $2-3 \mathrm{~mm}$ respectively.

\section{Discussion}

Gallstone ileus (GSI) is a rare cause of gastrointestinal obstruction. It accounts for $25 \%$ of mechanical small-bowel obstruction in patient over the age of 65 , with a mortality of $12-50 \%$. $^{1}$ The most frequent site of obstruction is the terminal ileum, ${ }^{2}$ it also may be found in the duodenum causing Bouveret's syndrome. ${ }^{3}$ Other obstruction points, including jejunum (30\%) and colon (2.5\%) may be seen. ${ }^{4}$ In our case, the impacted stone was found at the terminal ileum. The mechanism of obstruction is usually that the gallstone enters the intestinal tract through a fistula formed between the gallbladder and the duodenum, stomach or colon. The gallstone must be at least $2.5 \mathrm{~cm}$ in diameter to cause obstruction. We are not aware of any case in the literature where gallstone ileus, volvulus and intestinal perforation were described concomitantly. The first diagnostic test for suspected small bowel obstruction would be an abdominal radiograph. However, the classic Rigler's triad (pneumobilia, small bowel obstruction and gallstone) has been reported to be present in only $30 \%$ to $35 \%$ of cases, since most of the gallstones are radiolucent. ${ }^{5}$ If abdominal radiograph does not reveal the diagnosis, computed tomography will allow visualization of the fistula and level of obstruction. Treatment depends on the fitness of the patient while therapies should be involved including relief of the obstruction, cholecystectomy and repair of the fistula. About $7 \%$ of GSI have been shown to settle with conservative management. However, in the majority of cases, operative management is required. ${ }^{6}$ In our present case, the gallstones enter small intestine through the conjunction of the common bile duct and the duodenum; the possible mechanism of the volvulus is small intestine's severely incongruous movement resulting from the GSI, The small bowel dilatation of proximal obstruction, intestinal loop gravity and postural changes induce the volvulus via the axis of the obstruction site. The perforation is because of pressure necrosis of ileum wall. It is considerable to select operative management timely for a similar case.

\section{Conclusion}

Though GSI is a rare cause of small bowl obstruction, it can result in serious complications such as volvulus and intestinal perforation, Timely intervention with operative management may improve the prognosis. This case should be kept in mind in a case of intestinal obstruction.

\section{Acknowledgements}

None.

\section{Conflict of interest}

Author declares that there is no conflict of interest.

\section{References}

1. De Palma GD, Mastrobuoni G, Benassai G. Endoscopic removal of a gallstone obstructing the lower ileum. Dig Liver Dis. 2009;41(6):446.

2. Chatterjee S, Chaudhuri T, Ghosh G, et al. Gallstone ileus an atypical presentation and unusual location. Int J Surg. 2008;6(6):e55-e56.

3. Chang CW, Chuan Shih S, Lin SC, et al. Gallstone ileus: a disease easily ignored in the elderly. International Journal of Gerontology. 2008;2(1):18-21.

4. Zahid FE, Benjelloun el B, Ousadden A, et al. Uncommon cause of small bowel obstruction - gallstone ileus: a case report. Cases J. 2009;2:9321.

5. Gajendran M, Muniraj T, Gelrud A. A challenging case of gastric outlet obstruction (Bouveret's syndrome): a case report. J Med Case Rep. 2011;5:497.

6. Osman N, Subar D, Loh MY, et al. Gallstone Ileus of the Sigmoid Colon: An Unusual Cause of Large-Bowel Obstruction. HPB Surg. 2010;2010:153740. 